\title{
Analysis of saliency of facial features through psychophysical scaling
}

\author{
HIROSHI HOJO ${ }^{1}$ \\ Department of Educational Psychology, School of Education, Waseda Universily, Shinjuku-ku, Tokyo 160
}

\begin{abstract}
Sixteen side-view smiling faces having two defining properties of the left eye and a mouth which were drawn by arcs with varying curvatures were rank ordered by 24 university students according to their perceived intensities of smiling. The analysis by a nonmetric maximum likelihood scaling method has revealed (1) that the data are approximately described by an inverse logistic function of a physical measure defined as a weighted sum of eye and mouth curvatures, and (2) that eye is 1.39 times as important as mouth in perceiving the intensity of smiling of faces. Comparison between the relative importance of eye to mouth estimated from this analysis and that from the preceding work on front-view smiling faces suggests that the right and left eyes are "integrally" combined to contribute to the overall perceived intensity of smiling of faces.
\end{abstract}

Key words: psychophysical scaling, directional rank orders, Akaike's information criterion (AIC) statistic, dimensional combinations, face perception.

According to Shepherd, Davies, and Ellis' (1981) review of research work on cue saliency of facial features, the most consistent finding has been that hair is the most important single feature. Shepherd et al. (1981), however, suggest that this finding only reflects the fact that hair was the facial feature physically most heterogeneous among all the relevant features in experiments conducted in those researches. It would be quite natural that those features whose physical values considerably vary tend to be more heavily weighed in recognition or similarity judgment tasks. Therefore, the weights representing the relative importance of features could not meaningfully be compared with each other if they are estimated from the data in which physical values of defining properties of stimuli used are not well controlled.

The degree of salience of features might also change according to subjects or to methods by which data are obtained. In fact, Takane and Sergent (1983) have

1 The author wishes to express his gratitude to professor Tatsuro Makino of Waseda University for his invaluable advice and encouragement. found that the salience order of features changes according to the subjects and the types of data obtained. Indeed, these changes in importance of features are psychologically meaningful and worthy of investigation. However, in such studies we must be careful not to confound the truly meaningful factors with psychologically meaningless factors even if the latter appears to affect the relative importance of features to the same degree as the former. In order to find what is the true factor that determines the salience of features, therefore, it might be best, in the first place, to get rid of the biases due to the differences between features in the degree to which physical values of each feature are varied.

From this point of view Hojo (1987) attempted to compare the saliency of eye and that of mouth using a set of stimuli whose defining features are physically controlled. More specifically, Hojo (1987) scaled perceived intensities of smiling of 16 front-view faces which were constructed by combining four levels in each of the eye and mouth curvatures (see Fig. 1). The data obtained by the directional ranking method were analyzed by a psychophysi- 
cal function model which has a weight parameter representing the relative importance of eye to mouth in judgments of the degree of smiling. One of the main results was that eye is exactly twice as salient as mouth in the judgments of the degree of smiling of schematic faces employed.

A question then arises: How does the relative importance of eye to mouth change when side-view smiling faces each with only one eye (i.e., the left eye as it is observed in Fig. 1 (b)) are used as stimuli? We cannot simply predict that, because the side-view face has only one eye while the front-view face has two eyes, the weight for the side-view face will be one-half the value for the front-view face. This problem is not so simple, since little is known about how the impressions of the right eye and the left eye are combined to contribute to the overall perceived intensity of a smiling face.

Thus, the purpose of this article is not
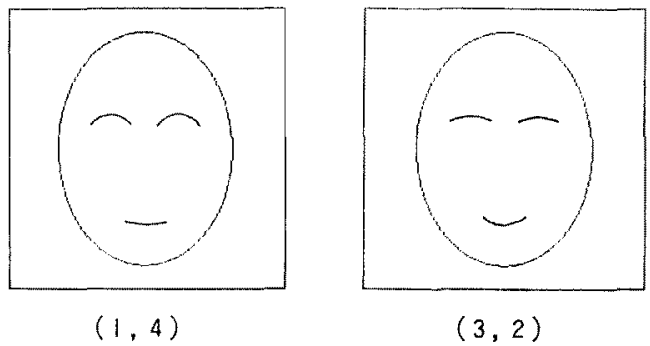

$(3,2)$

(a)

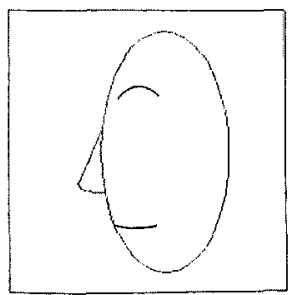

$(1,4)$

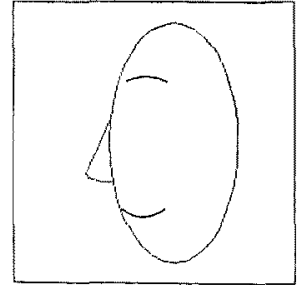

$(3,2)$ (b)

Fig. 1. Samples of front-view smiling faces in (a) and those of side-view smiling faces in (b). A pair of figures in each parenthesis are the levels of eye and mouth curvatures of the face. only to scale the judged intensities of smiling of side-view faces, but also to clarify the relation between the two respective weights estimated from the side- and frontview face data.

\section{Method}

Our general approach is in line with the maximum likelihood scaling method developed by Takane and his colleagues (e.g., Takane, 1978; Takane, 1981; Takane \& Garroll, 1981). According to their approach a set of three constituent models, the representation, error, and response models, are specified so that the likelihood of observed data may be stated in terms of parameters in these models. However, since the method adopted here is exactly the same as that in Hojo (1987) in which, as mentioned above, the judged intensities of smiling of front-view faces were scaled, only the representation models will be described in what follows (see Hojo, 1987, or Takane, 1981, for example, for the other two constituent models).

For the representation models of the perceived intensity of smiling we examine three distinct models designated as the psychophysical function model (PFM), the unconstrained model (UCM), and the quasi-psychophysical function model (QFM) (Hojo, 1985).

Let face $(i, j)$ denote a face with the $i^{\text {th }}$ eye curvature level and the $j^{\text {th }}$ mouth curvature level, and $x_{E_{i}}, x_{M}$, represent physical values of the $i^{\text {th }}$ and $j^{\text {th }}$ curvature levels of eye and mouth, respectively. (Note that symbol $x_{L}$, was used for $x_{M}$ in Hojo, 1987 , where the word "lips " was used in place of "mouth".) The PFM assumes that the judged intensity of smiling of face $(i, j), s_{i j}$, is a function of $y_{i j}$, which is defined as

$$
y_{i j}=w_{1} x_{E_{i}}+x_{M_{j}},
$$

where $w_{1}$ is a non-negative weight representing the relative importance of eye to mouth (the weight of 1 is tacitly applied to 
$\left.x_{M_{j}}\right)$.

As a form of the function we choose the following inverse logistic function:

$$
s_{i j}=-\frac{1}{A} \ln \left(\frac{B}{y_{i j}}-1\right)+C, \quad A, B>0,
$$

where $A$ and $C$ are unspecified parameters, namely the unit and the origin of the scale, and $B$ is an estimable parameter, the position of a point along the measure $y_{i j}$ at which $s_{i j}=\infty ; s_{i j}$ increases very rapidly as $y_{i j}$ approaches this point.

There are four points to be noticed as regards this PFM: First, the model (2) states that changes in the lower and upper ranges of $y_{i j}$ are steep, and that in the middle ranges of $y_{i j}$ the slope is less steep; we choose (2) rather than a linear function model $\left(s_{i j}=A y_{i j}+G\right)$ because the former was found the better than the latter by pilot studies. Second, the weight parameter $w_{1}$ is directly applied to physical value $x_{E_{i}}$, which makes this weight parameter free from the difficulties discussed at the outset of the paper. Third, two physical values $x_{E_{i}}$ and $x_{M}$ are assumed to be linearly combined into physical measure $y_{i j}$. Fourth, $w_{1} x_{E_{i}}$ and $x_{M}$ are the respective contributions of eye and mouth to the overall physical measure $y_{i j}$, but it would be justifiable to assume that they also represent the contributions of these features to $s_{i j}$ because $s_{i j}$ is defined as a strictly increasing function (the inverse logistic function) of $y_{i j}$, though their contributions to $y_{i j}$ are not precisely the same as those to $s_{i j}$. Hence, the $w_{1}$ parameter approximately reflects the relative importance of eye to mouth in the judgments of the degree of smiling.

Now, the UCM is merely a set of scale values, usually expressed as a vector of scale values such as $\boldsymbol{s}=\left(s_{11}, \ldots, s_{\mathbf{4 4}}\right)$ in the present example. Since the UCM is thus the least restrictive representation model, we may ask a question about the adequacy of the other representation models against this model. In this model we usually impose a restriction $s_{i j}=s_{k l}$ for any
Table 1

Summary of the results obtained by fitting the three representation models to the data

\begin{tabular}{lcc}
\hline $\begin{array}{c}\text { Representation } \\
\text { models }\end{array}$ & $\begin{array}{c}\text { Numbers of free } \\
\text { parameters for } \\
\text { the models }\end{array}$ & AIC \\
\hline UCM & 10 & 1068.1 \\
PFM $\left(w_{1}=1.36\right)$ & 3 & 1081.4 \\
QFM $\left(w_{1}=1.39\right)$ & 8 & 1061.2
\end{tabular}

$i, j, k$, and $l$ if they are close enough to avoid overparametrization. The QFM, a special case of the UCM, consists of the above PFM (2) and a term representing interaction effects presumed to exist between curvatures of the eye and the mouth; that is, it is written as $s_{i j}=f\left(y_{i j}\right)+c_{i j}$, where $f\left(y_{i j}\right)$ corresponds to the PFM given above by (2), and $c_{i j}$ is the interaction effects between the $i^{\text {th }}$ and $j^{\text {th }}$ levels of eye and mouth curvatures, respectively. It is again assumed that $c_{i j}=c_{k l}$ if they are close enough. We consider this model because the preceding study for the front-view face data has suggested that this model is the most appropriate model. (More detailed descriptions of these two models are available in Hojo, 1985, 1986.)

\section{Results of Application and Discussion}

We obtained the directional rank order judgments from 24 students according to the intensity of smiling of 16 side-view faces. These stimuli were constructed in the manner similar to the one in which the front-view faces were constructed (see Fig. 1).

The three representation models, the UGM, the PFM, and the QFM were fitted to the data. In order to compare the goodness of fit of these models we used the AIC statistic (Akaike, 1974) in this analysis. The main results are presented in Table 1 and in Fig. 2. According to the $\mathrm{AIC}$ criterion (the smaller it is, the better is the fit) the QFM is found to be the most appropriate model among the three.

We may say that although the UGM 
fits the data better than the PFM, the PFM is still a fairly good model of the intensity of smiling of a face because the PFM in the QFM is considered to account for a large portion of variability (see Fig. 2), but that the interaction effects between the two features $\left(c_{i j}\right)$ cannot be disregarded to explain all meaningful variations in the data. The above is quite consistent with the result of the analysis of the front-view face data.

\section{Interpretations of the Weight}

The estimate of the weight parameter, $w_{1}$, indicates (see Table 1) that the eye contributes to the overall physical measure $y_{i j} 1.39$ times as much as the mouth, implying that the eye is 1.39 times as important as the mouth in perception of the intensity of smiling of, at least, such faces as have their eyes and mouths characterized by varying curvatures alone.

As mentioned earlier, an interesting question is what relations underlie between this weight value of 1.39 and that of 2.00 estimated from the front-view face data

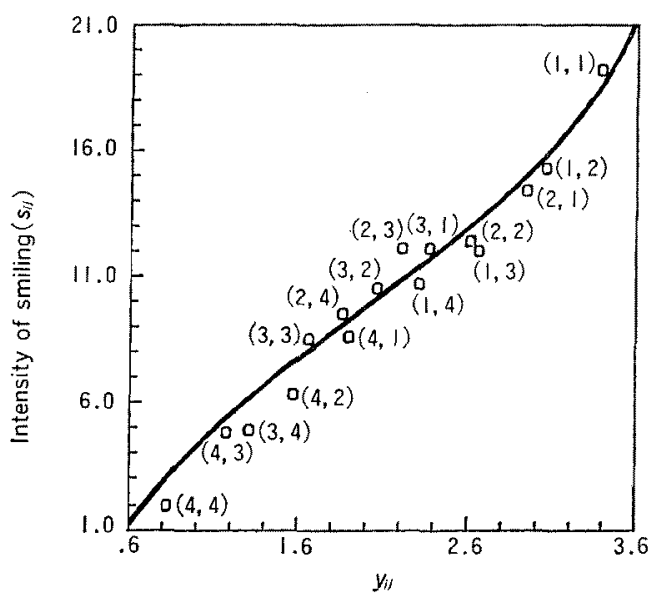

Fig. 2. The perceived smiling intensities of 16 schematic faces predicted from the QFM are plotted as a function of physical measure $y_{i j}$. The curve in the graph represents the predictions from the PFM $\left(s_{i j}=(-1 / .20) \ln \left(3.92 / y_{i j}-1\right)+9.58\right)$ in this QFM, A pair of figures in each parenthesis are the levels of eye and mouth curvatures of the face.
(Hojo, 1987).

Let us approach this problem from a viewpoint of dimensional combinations in a processing system for multidimensional objects. Garner (1974) distinguishes several types of dimensional combinations or structures, but the two which are particularly relevant in the present analysis are those dimensional combinations that are integral and those that are separable. According to Garner (1974) dimensions are integral if it is impossible to specify the value of one dimension without simultaneously specifying the value of the other dimension, while they are said to be separable if it is possible to do so. (See Garner, 1974, or Spoehr and Lehmkuhle, 1982, for more comprehensive accounts.)

As an example, consider dissimilarity ratings among objects each defined by two dimensions. It may be that the dissimilarity rating is well described by the Euclidean metric, namely by the straight-line distance between two objects placed in a two dimensional space if the two dimensions are integrally combined, because then the individual dimensions are not immediately apprehended or perceived. On the other hand, if they are separable dimensions, the dissimilarity rating might be well described by the city-block metric, namely the sum of the distance between two objects on one dimension and that on the other, since then the individual dimensions are perceived immediately and independently of one another (Spoehr \& Lehmkuhle, 1982). Formally the Euclidean metric, $d_{X Y}{ }^{E}$, and the city-block metric, $d_{X Y}{ }^{C}$, are given by

$$
d_{X Y} E=\left(d_{X}^{2}+d_{Y}^{2}\right)^{1 / 2}
$$

and

$$
d_{X Y}{ }^{C}=d_{X}+d_{Y},
$$

respectively, where $d_{X}$ and $d_{Y}$ are the distances between two objects on dimensions $X$ and $Y$, respectively.

Thus, if we apply this viewpoint of dimensional combinations to the present 
problem, our question is restated as follows: If the contribution of the right eye and that of the left eye are integrally combined to produce the overall contribution of a pair of eyes to physical measure $y_{i j}$, letting the contribution of the mouth be $x_{M_{j}}$, then the combined contribution of the two eyes with the $i^{\text {th }}$ curvatures is to be predicted from the Euclidean metric assumption

or

$$
\begin{aligned}
w_{2} x_{E_{i}} & =\left\{\left(w_{1} x_{E_{i}}\right)^{2}+\left(w_{1} x_{E_{i}}\right)^{2}\right\}^{1 / 2} \\
& =\sqrt{2} w_{1} x_{E_{i}}
\end{aligned}
$$

$$
w_{2}=\sqrt{2} w_{1} .
$$

If they are separable, it must be predicted by the city-block assumption

that is,

$$
\begin{aligned}
w_{2} x_{E_{i}} & =w_{1} \lambda_{E_{i}}+w_{1} x_{E_{i}} \\
& =2 w_{1} x_{E_{i}}
\end{aligned}
$$

$$
w_{2}=2 w_{1} .
$$

Substituting our estimates $w_{1}=1.39$ and $w_{2}=2.00$ into equations (3) and (4) reveals that the observed value of 2.00 for the two-eye condition is very close to the value of $1.97(=\sqrt{2} \times 1.39)$ predicted under the integral combination assumption. We are thus led to the conclusion that the contributions of right and left eyes to $y_{i j}$, and hence approximately $s_{i j}$ as noted earlier, are integral in the sense of Garner (1974) at least for the present data.

Finally, we would like to point out that the above question thus far has been discussed without considering the interaction effects between the two features. If they are taken into account in the discussion, the problem may become more complicated from a theoretical point of view, but it would be beyond the scope of the present study to go into this problem.

\section{Concluding Remarks}

The present conclusion about the combination of contributions of the two eyes is in agreement with our intuition that " a pair of eyes " are something different from a simple addition of the two eyes. It must be noted, however, that this finding is obtained from the judgments of the intensity of smiling. Therefore, from this result alone it does not necessarily follow that our hypothesis of integral combination of right and left eyes holds in the perception of faces in general. It would be interesting and worthwhile to study further this subject from different directions, such as scaling of similarities among faces, for example.

\section{References}

Akaike, H. 1974 A new look at the statistical model identification. IEEE Transactions on Automatic Control, 19, 716-723.

Garner, W. R. 1974 The processing of information and structure. Potomac, Md.: Lawrence Erlbaum Associates.

Hojo, H. 1985 Scaling of dot dispersion from directional rank order data. Japanese Psychological Research, 27, 1-10.

Hojo, H. 1986 Psychophysical scaling of pentagons. Japanese Psychological Research, 28, 11-20.

Hojo, H. 1987 Psychophysical scaling of smiling faces. Japanese Psychological Research, 29, 37-41.

Shepherd, J,, Davies, G., \& Ellis, H. 1981 Studies of cue saliency. In G. Davies, H. Ellis \& J. Shepherd (Eds.), Perceiving and remembering faces. New York: Academic Press. Pp. 105-131.

Spoehr, K. T., \& Lehmkuhle, S. W. 1982 Visual information processing. San Francisco: Freeman.

Takane, Y. 1978 A maximum likelihood method for nonmetric multidimensional scaling: I. The case in which all empirical pairwise orderings are independent-theory. Japanese Psychological Research, 20, 7-17.

Takane, Y. 1981 Multidimensional successive categories scaling: A maximum likelihood method. Psychometrika, 46, 9-28.

Takane, Y., \& Carroll, J.D. 1981 Nonmetric maximum likelihood multidimensional scaling from directional rankings of similarities. Psychometrika, 46, 389-405.

Takane, Y., \& Sergent, J. 1983 Multidimensional scaling models for reaction times and same-different judgments. Psychometrika, 48, 393-423.

(Received Sept. 19, 1985; accepted March 14, 1987) 